\title{
INTRACELLULAR LUCIFER YELLOW INJECTION IN FIXED BRAIN SLICES COMBINED WITH RETROGRADE TRACING, LIGHT AND ELECTRON MICROSCOPY
}

\author{
E. H. BUHL ${ }^{*} \ddagger$ and J. LüBKE†§ \\ *Max-Planck-Institut für Hirnforschung, Deutschordenstrasse 46, 6000 Frankfurt 71, F.R.G. \\ †Max-Planck-Institut für Biophysikalische Chemie, Am Fassberg, 3400 Göttingen, F.R.G.
}

\begin{abstract}
The present paper contains a full methodological description of iontophoretic Lucifer Yellow injections in fixed brain slices in mammals. In brief, cortical tissue was either perfused or immersion-fixed in paraformaldehyde. After Vibratome sectioning, tissue slices were transferred to epifluorescence microscopes equipped with long distance objectives. Under visual guidance, neurons were selectively impaled with Lucifer Yellow-filled electrodes and intracellularly injected until all dendrites appeared brightly fluorescent. Excellent dendritic staining was obtained in both perfusion-fixed cat visual cortex and immersion-fixed human brain biopsies. Dendritic spines, varicosities and growth cones could be readily discerned. Filling of axonal collaterals was, however, incomplete.

Callosally projecting neurons in cat visual cortex were retrogradely traced with a mixture of the fluorescent tracers Fast Blue and Dil. Subsequently the morphology of labelled cells was determined by intracellular Lucifer Yellow injection. Although the Fast Blue fluorescence had become undetectable in filled neurons the granular red appearance of Dil was still discernible. Hence the neuronal composition of even rełatively sparse projections can be demonstrated. To obtain permanent preparations, dye-filled neurons were immersed in a diaminobenzidine solution and irradiated with epifluorescent illumination until all visible fluorescence had faded. Photo-oxidation resulted in the intracellular formation of a homogeneously distributed brown reaction product visible with the light microscope. Brief osmication enhanced the staining contrast, thus providing a Golgi-like image. Subsequent electron microscopy of photo-converted cells showed the fine granular nature of the electron opaque reaction product, thus revealing numerous cytological features. The precipitate was homogeneously distributed throughout the entire cytoplasm and nucleus, extending into dendrites and axon. Any apparent leakage of the label into the extracellular space was not observed.

Intracellular staining in fixed tissue yields a high number of neurons with extensive filling of dendritic arbors. Photo-oxidation provides stable, non-fading preparations with the option of subsequent electron microscopy. In addition, the technique can be combined with immunocytochemistry and a variety of fluorescent tracer substances. These features, combined with its high selectivity and relative methodological simplicity, render the method to be a promising alternative to classical neuroanatomical approaches.
\end{abstract}

Intracellular staining in vivo has become well established as a powerful neuroanatomical tool, particularly with respect to the morphological and pharmacological analysis of functionally characterized neuronal elements. ${ }^{25,26,39,43,44,50,66,71}$ The tracer horseradish peroxidase (HRP) and various cobalt methods provide excellent results with both the light and electron microscope. ${ }^{2,18,24,36-38,54}$ Due to the relatively low quantum yield and low electron density many fluorescent dyes, such as Procion Yellow, are in many respects inferior to these tracers. ${ }^{69}$ During exposure to ultraviolet light, fluorescent markers such as carboxyfluorescein show high susceptibility to photo-

¡Present address: Vision, Touch and Hearing Research Centre, Department of Physiology and Pharmacology, University of Queensland, St. Lucia, Queensland 4067, Australia.

§To whom correspondence should be addressed.

Abbreviations: DAB, 3,3'-diaminobenzidine; DAPI, 4,6-diamidino-2-phenyl-indol; HRP, horseradish peroxidase; LY, Lucifer Yellow; PB, phosphate buffer; PN, postnatal oxidation. ${ }^{55}$ Furthermore, the latter dye is lost from the tissue during fixation and dehydration procedures. Lucifer Yellow (LY), however, due to its intense fluorescence, long stability during illumination and its compatibility with most embedding procedures, has been successfully used in a variety of biological systems. ${ }^{69,70}$ Although LY itself has no significant electron density, a relatively simple protocol can transfer the dye into a stable, fine granular electron-dense reaction product, therefore facilitating fine structural analysis of filled neurons. ${ }^{49,79}$

Intracellular work in the living preparation requires appropriate electrophysiological facilities. Moreover, the yield of successfully penetrated, and subsequently stained, cells is usually too low to consider the method useful for quantitative studies. In the mammalian nervous system in particular there are problems of limited accessibility to many regions, and of biased samples due to the relative frequency distribution of different neuronal types and/or small soma sizes of some cell classes. These problems have been resolved by the availability of visually controlled 
iontophoresis of $L Y$, recently demonstrated by injections into prelabelled amacrine cells in aldehyde-fixed rabbit retinae. ${ }^{72.73}$ Since fixed tissue may be used over long periods, the yield of cells is significantly increased. In addition, visually guided penetration of prelabelled cells offers a high degree of selectivity, superior to most other neuroanatomical staining methods.

The present paper aims to provide an extensive methodological description of LY injection in aldehyde-fixed slices of mammalian cortex, and to describe the combination of this technique with other neuroanatomical methods.

\section{EXPERIMENTAL PROCEDURES}

\section{Surgery and perfusion}

The visual cortex of 10 cats ranging from newborn to adult was investigated in a developmental study of layer VI pyramidal cells. All animals were maintained from birth (postnatal day 1; PN 1) in a $12 \mathrm{~h}$ light-dark cycle. After deeply anaesthetizing the cats with sodium pentobarbitone (Nembutal ${ }^{\mathrm{N}}, 60 \mathrm{mg} / \mathrm{kg}$ body weight, i.p.), they were perfused through the heart or ascending aorta. Phosphate buffered $(0.05 \mathrm{M}$ phosphate buffer (PB), pH 7.4) physiological saline containing Heparin (Liquemin ${ }^{\text {th }}, 25,000$ I.U.) was used to rinse the vascular system. When the washing fiuid appeared to be clear the perfusion was continued over $2045 \mathrm{~min}$ with $114 \%$ paraformaldehyde in $0.1 \mathrm{~PB}, \mathrm{pH} 7.4$. For better post-fixation the brain was usually left in situ for the next $2 \mathrm{~h}$ and sometimes overnight post-fixation in the same fixative at $4^{\circ} \mathrm{C}$ was required for the relatively soft brains of younger animals. Experiments now in progress show that addition of $0.1-0.5 \%$ glutaraldehyde for subsequent use in electron microscopy does not impede the staining quality.

Small pieces of human cortex were obtained from brain biopsies which had been surgically removed for medical reasons, usually malignant brain tumours (for further details about the material see Table $I$ in Buhl and Schlote ${ }^{8}$ ). Roughly $\mathrm{I} h$ after removal the tissue had been immersed in $4 \%$ paraformaldehyde in $0.1 \mathrm{M} \mathrm{PB}$ at $\mathrm{pH} 7.4$. After an initial inspection, tissue which appeared normal macroscopically was separated and further immersed for $3-4 \mathrm{~h}$ in the same fixative. Prior to sectioning the cortex was stored at $4 \mathrm{C}$. The only material included for analysis was that which on subsequent inspection with the light microscope was shown to be anatomically normal.

Three adult cats were used for the combined retrograde tracing and intracellular injection of callosally projecting cells in visual cortex. The animals were anaesthetized with an intramuscular injection of $30 \mathrm{mg} / \mathrm{kg}$ of ketaminehydrochloride (Ketanest ${ }^{\mathrm{k}}$, Parke Davis) and $15 \mathrm{mg} / \mathrm{kg}$ of xylazinehydrochloride (Rompun ${ }^{\text {* }}$, Bayer). For subsequent doses of the same anaesthetic an intravenous catheter was inserted transcutaneously into an antebrachial vein. Then the animal was placed in a stereotaxic headholder. A large craniotomy was performed to expose visual areas 17 and 18 of the right hemisphere. After the dura mater had been cut, several large injections (up to $10 \mu \mathrm{l}$ ) of a mixture of equal amounts of the fluorescent tracer Fast Blue ${ }^{3}$ (Dr. Illing GmbH, GroB-Umstadt, F.R.G.; 5\% dissolved in distilled water) and the carbocyanine dye Dil ${ }^{10.29 .74}$ (Molecular Probes, Eugene, OR; $5 \%$ dissolved in a mixture of $60 \%$ distilled water, $30 \%$ ethanol and $10 \%$ dimethylsulphoxide) were made with a $10 \mu 1$ Hamilton syringe around the vertical visual meridian. The position of the latter had been roughly determined by comparison with published maps. ${ }^{75}$ After the injections were completed the dura was sealed and the piece of skull reinserted and stabilized with dental cement. Then the skin was sutured and the animals were allowed to survive under antibiotic treatment for 46 days.

\section{Tissue preparation for intracellular injection}

A piece of cortex was blocked with a non-toxic glue (Histoacryl) and without further embedding serially cut on a Vibratome (Oxford Instruments, U.K.; or Dosaka Instruments, Tokyo, Japan). Blocks of cortex and slices not required for immediate injections were stored in $0.1 \mathrm{M} \mathrm{PB}$ (pH 7.4) in the refrigerator. Thickness of sections ranged from 100 to $200 \mu \mathrm{m}$, since injections in thinner slices revealed too little of the dendritic arbors. Thicker sections, however, increased background fluorescence. In this material excellent staining quality was obtained for periods of up to 12 days

Prior to mounting for LY injection, the slices from cat visual cortex which were used to study layer VI pyramidal cells were incubated for $5-10 \mathrm{~min}$ in a $10^{-7} \mathrm{M}$ aqueous solution of the DNA stain 4,6-diamidino-2-phenyl-indol (DAPI, Sigma). This staining procedure resulted in brightly fuorescent nuclei which rendered a suitable target for the LY-filled pipettes (Fig. ID) and also determined the layer VI/white matter border (Fig. 1C).

For LY injection, slices were carefully mounted, with a brush, onto a slide. The tissue was held down by a fenestrated Millipore filter, the aperture being slightly smaller than the slice. Small pieces of metal were placed on the filter to prevent floating of the slice in the injection chamber (Fig. 1B). Finally the preparation was immersed in a petri dish containing $0.1 \mathrm{M} \mathrm{PB}$ at room temperature. The injection chamber was attached to a plexi glass plate which fitted the slide holder of the microscope stage (Fig. 1B). Addition of ascorbic acid to the chamber fluid, originally used to oxidize $L Y$ which leaked into the bathing solution, ${ }^{72}$ had little effect in suppressing background staining and was therefore omitted in later experiments.

\section{Microscope and micromanipulators}

Due to the absence of electrophysiological criteria to monitor the successful penetration of neurons, epiffuorescence illumination (Zeiss filter combination for Lucifer Yellow: BP 400 440, FT 460, LP 470) was essential to visually guide the pipette and control dye injection. Inverted microscopes (as used for patch clamp recording or slice electrophysiology) have the major disadvantage that the uncleared slice, due to its location between the electrode tip and the objective, obscures the optics.

Focussing with conventional epifluorescence microscopes is achieved by vertical stage movements. Therefore, if a micromanipulator were attached to the microscope base this would result in undesired position shifts of LY pipettes and tissue. Trying to focus on the slice while having the fuorescent electrode tip already in focus results in elevating the stage and simultaneously piercing the pipette into the tissue. Two different, but equally successful, strategies solved the problem. Firstly, attachment of a small manipulator to the stage of a modified Zeiss Unimat microscope results in a static spatial arrangement of electrode and tissue (Fig. IA). Alternatively the choice of the Zeiss ACM, due to its construction as a fixed stage microscope, creates the same effect.

For adequate positioning of the micropipettes a large working distance, particularly at higher magnifications, proved to be advantageous. Zeiss (UD 20/0.57, UD 40/0.65) and Nikon $(E L W D \times 40)$ long distance objectives with working distances ranging from 8 to $10 \mathrm{~mm}$ allowed an electrode position approximately $40^{\circ}$ from the vertical plane. Intracellular penetration in electrophysiological slices frequently requires high speed stepping devices, which, however, guarantee sufficient precision only in one spatial orientation. Tauchi and Masland, ${ }^{72}$ in previous work on fixed retinal flat mount preparations, already demonstrated that paraformaldehyde fixation stabilizes and hardens cellu- 

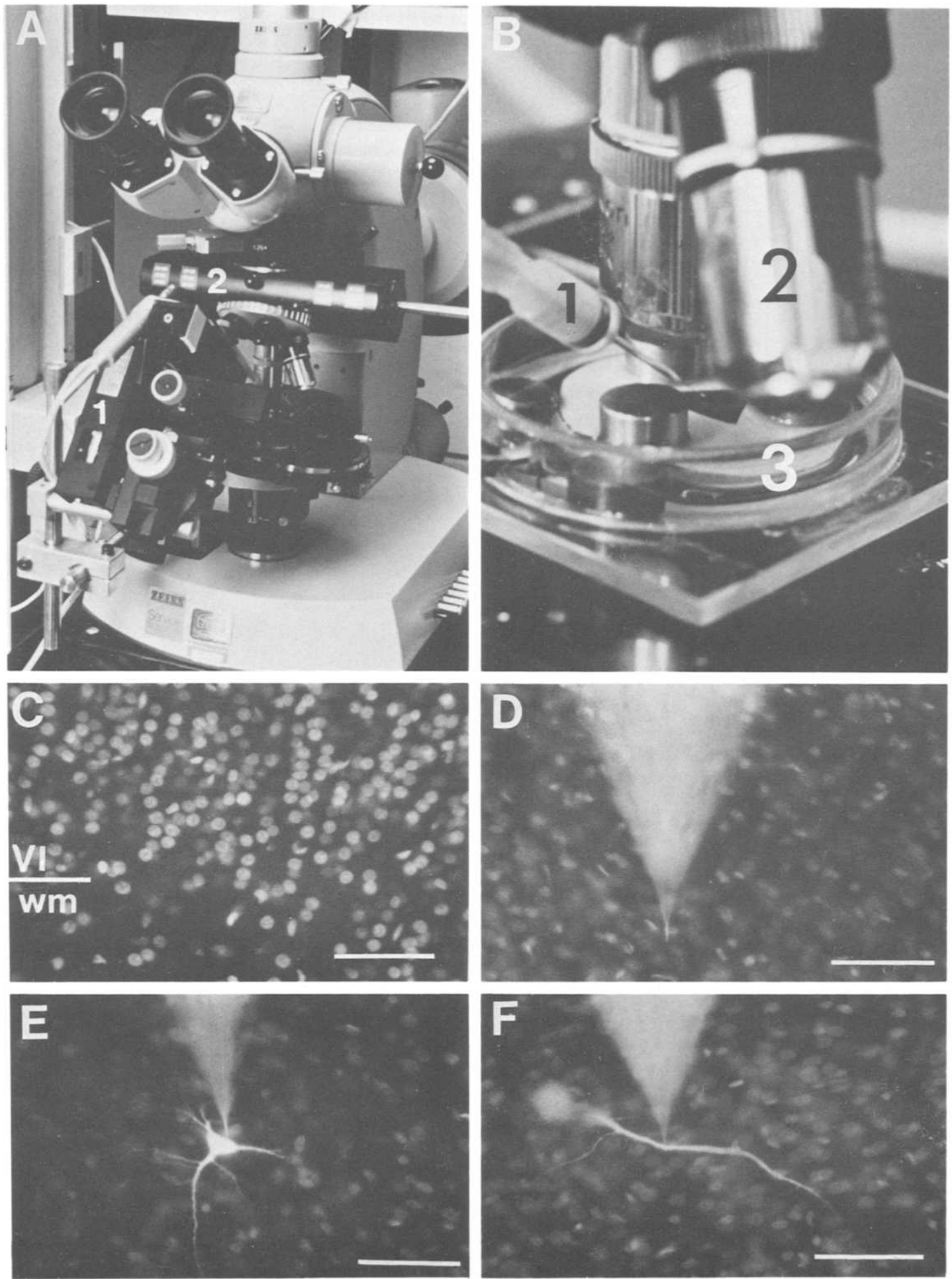

Fig. 1. (A) Modified Zeiss Unimat epifuorescence microscope (2) used for intracellular LY injections in fixed slices. The micromanipulator (I) is attached to the microscope stage. (B) Detail of (A) showing the electrode holder (1), objectives (2) and slice chamber (3). A slice is held in place by small metal discs on a fenestrated Millipore filter. (C) Slice of cat visual cortex after $10 \mathrm{~min}$ incubation in a $10^{-7} \mathrm{M}$ DAPI solution. The layer VI/white matter (wm) border is sharply demarcated by a decrease in the density of fluorescent nuclei. (D) LY-filled micropipette positioned over the slice surface. Cytoarchitectonic boundaries as revealed by the nuclear DAPI-stain served to guide the electrodes. (E, F) LY-filled cells which had been injected through the soma (E) or the apical dendrite (F). Scale in (C)-(F) $50 \mu \mathrm{m}$. 
lar membranes sufficiently to circumvent the use of high speed stepping drives. Therefore, hand or motor driven high precision mechanical manipulators which could be moved in three axes were chosen. Three different manipulators were tested and considered to be equally useful: two micromanipulators from Märzhäuser KG (Wetzlar, F.R.G.), one equipped with three electro-motors and joystick (Neuroscience Trading, Frankfurt, F.R.G.); a Huxlcy-type micromanipulator (Goodfellows Lid, England) rendered results of comparable quality.

\section{Pipette puller and electrodes}

Both horizontal (Narashige Instruments, Tokyo, Japan) and vertical electrode pullers (Kopf Instruments, Tujunga, CA, U.S.A.) provided adequate pipettes. Two different types of omega dot capillaries were employed: glass capillaries (Clark Electromedical Instruments; $1.0 \mathrm{~mm}$ o.d. $\times 0.86 \mathrm{~mm}$ i.d.) and thick walled borosilicate tubing (F. Hilgenberg, Malsfeld, F.R.G.; $1.2 \mathrm{~mm}$ o.d. $\times 0.69 \mathrm{~mm}$ i.d.). To produce very fine tips with a long shank, filament heat and solenoid were adjusted accordingly (for details see Brown and Flaming ${ }^{5}$ ). The resistance of the microelectrodes, measured in $0.1 \mathrm{M}$ PB, usually ranged between 90 and $400 \mathrm{M} \Omega$. Despite the relatively high resistance, pipettes normally did not block when currents $<3 \mathrm{nA}$ were applied. The fine tip sizes rendered it unnecessary to bevel or break tips to facilitate intracellular injection.

\section{Injection procedure}

After mounting the slice in the injection chamber the latter was transferred to the stage of the epifluorescence microscope. Fluorescing neurons could be readily identified in all preparations due to either retrograde labelling with Fast Blue, nuclear staining with DAPI (Fig. 1C-F) or the content of autofluorescing age pigment lipofuscin in the human material. Pipettes filled with a 3-5\% aqueous solution of LY were aimed at fluorescing target neurons in the depth of the slice preparation (Figs $1 \mathrm{C}-\mathrm{F}$ and $3 \mathrm{~A}$ ). When the tip of the LY pipette made contact with the soma membrane a bright yellow dot was quite often visible at the tip and an increase of the pipette resistance was measured. If a slight forward movement of the electrode did not result in membrane penetration, this could be facilitated by gently tapping the stage of the microscope or the micromanipulator. When a small pulse of negative current was subsequently applied, rapid and intensive dye filling of the cell body was monitored (Fig. IE). If, however, the electrode was still located in the extracellular space LY quickly diffused.

After successful impalement of a cell body (Figs IE and 3A) or a primary dendrite (Fig. 1F) dye was injected with a constant negative current of 1-3 nA for 3-10 min. Application of pulsed currents did not improve the staining quality. Dye filling of a cell was judged complete when fine distal processes appeared brightly fluorescent (Fig. 2A-D). Gradual tapering of the staining intensity towards dendritic tips indicated understaining.

In experiments where callosally projecting neurons had been retrogradely labelled from the contralateral visual cortex, the Fast Blue component of the tracer mixture served as a strong marker, being visible in the LY-filter combination. Due to the intense brightness of LY, however, the Fast Blue label rapidly became undetectable. Therefore, switching to the rhodamine filter, where LY fluoresces very faintly, revealed the cytoplasmic content of bright red, granular Dil-label (Fig. 3B, D).

\section{Photo-oxidation and embedding}

Virtually all fluorescent dyes are susceptible to gradual fading when illuminated at their excitation wavelengths. Even the use of relatively stable substances such as LY and the addition of various non-fading reagents to the embedding media does not completely abolish this problem.
Prolonged storage of the tissue may result in gradually increasing background autofluorescence. To overcome these problems and obtain a permanent record of the filled cells stained and glycerol embedded neurons can be documented in serial photographs. ${ }^{7.16}$ Photographs with different planes of focus were taken with Zeiss Neofluar objectives on high speed black and white films, which provided the basis for subsequent graphical reconstruction by means of projected and superimposed negatives. However, if high resolution of fine morphological details is required the latter approach requires a large amount of film.

To study developmental features of layer VI pyramidal neurons in cat visual cortex, optimal optical resolution was required. Therefore, to transform $L Y$ into a stable and light microscopically visible reaction product, slices with $L Y$ filled neurons were preincubated for $5-10 \mathrm{~min}$ in $0.1 \mathrm{M} \mathrm{PB}$ (pH 7.4) containing $1.5 \mathrm{mg} / \mathrm{ml}$ diaminobenzidine Grade II (DAB, Sigma). After filled cells had been irradiated for 20-30 min with the LY excitation wavelengths all visible fluorescence had faded, resulting in the formation of a homogeneously brown reaction product contained within perikarya and dendrites. Excess DAB was washed out with several rinses in PB. Then the sections were transferred to distilled water and dried on slides. To enhance the staining contrast, slices were briefly $(2 \mathrm{~min})$ post-fixed with a $1 \%$ osmium tetroxide solution in $0.1 \mathrm{M} \mathrm{PB}$. This was followed by dehydration in an ascending series of ethanol, clearing in xylene and embedding in resin. Due to the highly osmiophilic nature of the DAB reaction product photooxidized pyramidal neurons appeared intensely black with only moderately increased background (Figs 4 and 5).

\section{Electron microscopy}

To determine the usefulness of the photo-converted material for ultrastructural analysis photo-oxidized human layer $\mathrm{V}$ pyramidal cells and cat layer VI pyramidal cells were processed for electron microscopy. Following the bleaching procedure, as described above, the tissue was post-fixed in a mixture containing $2 \%$ paraformaldehyde and $0.5 \%$ glutaraldehyde (in $0.1 \mathrm{M} \mathrm{PB}, \mathrm{pH} 7.4$ ) for $10 \mathrm{~min}$, blockstained with uranyl acetate in $50 \%$ ethanol. After dehydration in an ascending series of either ethanol or acetone, sections were embedded in Araldite or Epon. Photo-oxidized neurons were readily identified in serial $1 \mu \mathrm{m}$ semithin, Toluidine Blue stained sections. Adjacent ultrathin sections were cut with a Reichert Um03 ultramicrotome and stained with Reynold's reagent (lead citrate). The material was analysed with a Zeiss EM 10 electron microscope.

\section{RESULTS}

\section{Intracellular injection}

When injected into neurons in fixed slice preparations (Fig. 2A-D) LY effectively stains the entire dendritic tree and remains confined within the cellular boundaries without any evidence for dye coupling through gap junctions into adjacent cells. 12,27,40,69 Higher magnification in the glycerol embedded preparations revealed that the dye had spread into terminal dendrites, spines, the main axon and the proximal portion of axon collaterals. Incompletely filled material was recognized by weaker fluorescence and the diminished intensity of staining intensity towards distal portions of the neuron. Only neurons where dendritic staining was considered complete and with a large fraction of the dendritic tree contained within the slice were included in the sample $( \pm 50 \%)$. Due to the absence of other stained elements in the 

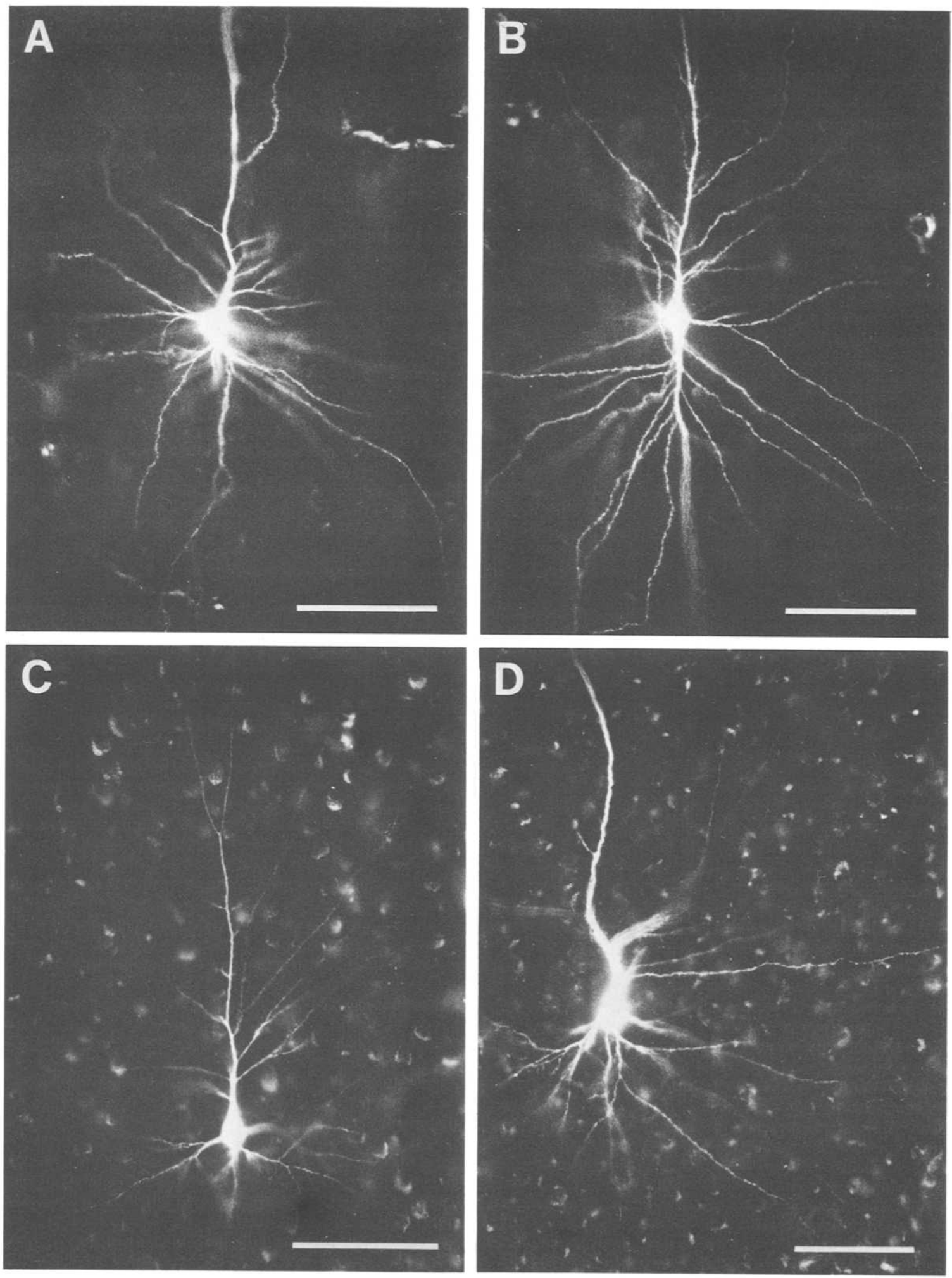

Fig. 2. A sample of spiny neurons in immersion fixed human cortex after intracellular LY injection. Due to the low magnification it is impossible to resolve dendritic spines. Without tapering towards the tips all dendrites reveal extensive dye filling, thus providing a Golgi-like impression. Depending on the age of the individuals varying amounts of the autofluorescent age pigment lipofuscin can be recognized in the background. (A) Layer III pyramidal cell. (B) Spiny, bitufted layer V neuron with pyramidal-like branching features. (C) Layer III pyramidal cell. (D) Layer VI pyramidal cell with highly asymmetric dendritic distribution. Scale in (A)-(D): $100 \mu \mathrm{m}$. 

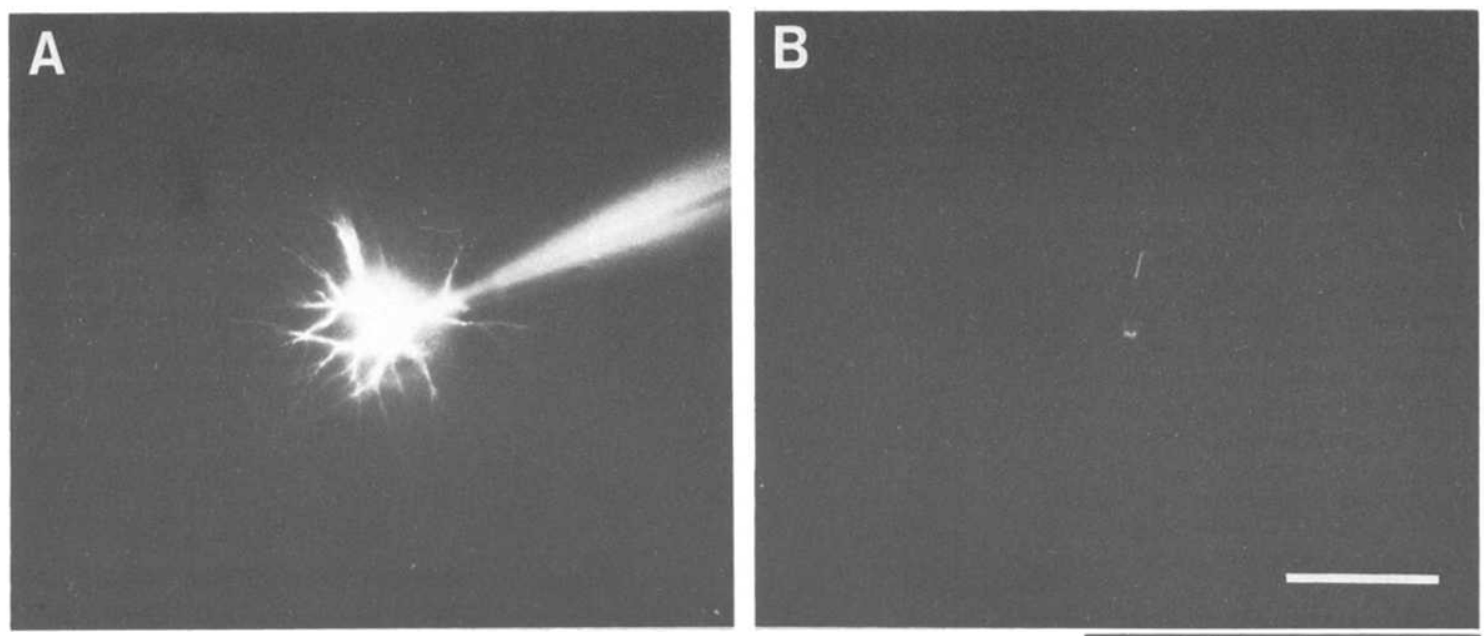

C

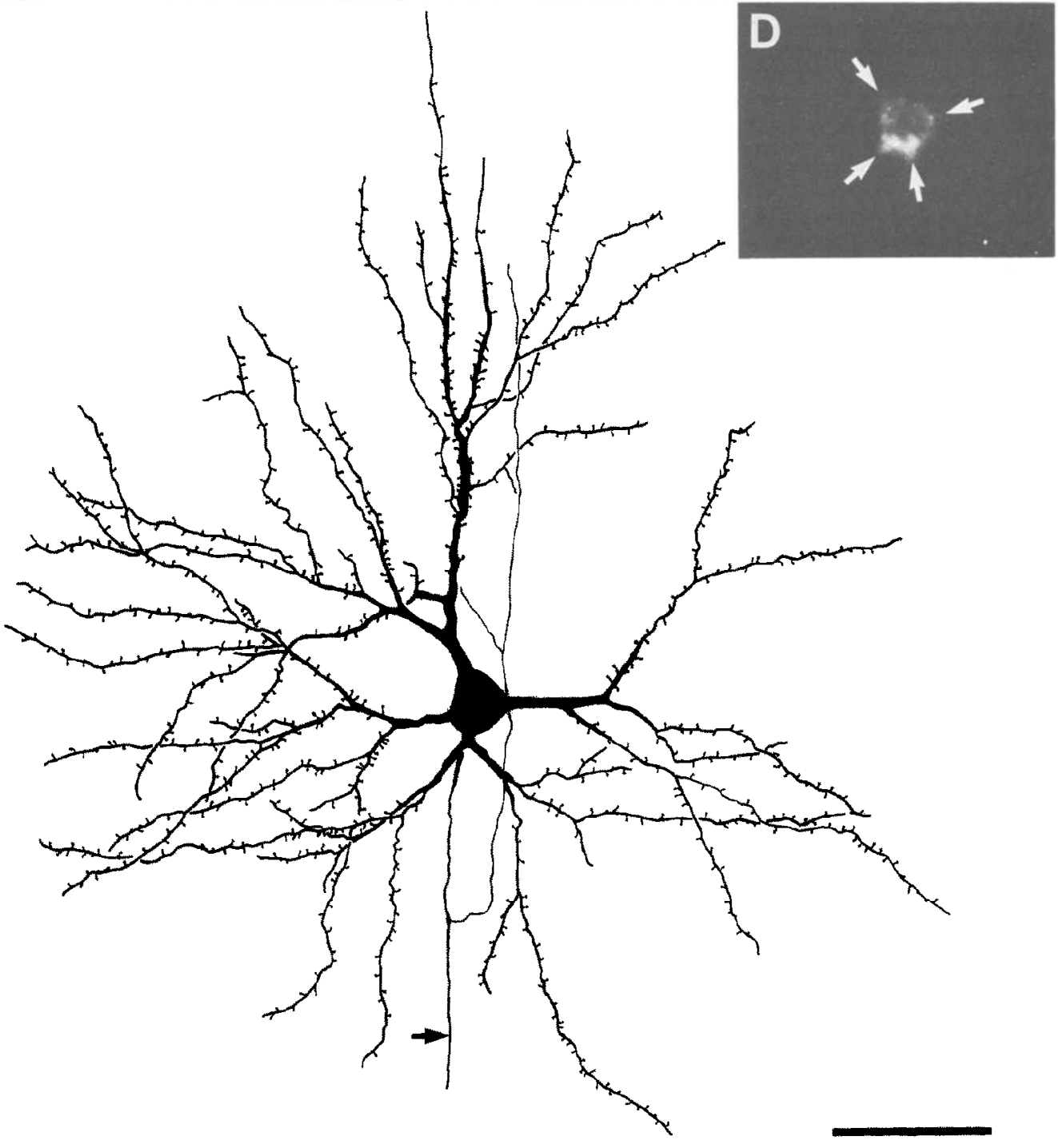

Fig. 3. Intracellular injection of tayer 11 pyramidal cell in cat visual cortex. Prior to injection the neuron had been retrogradely labelled with a mixture of the fluorescent dyes Fast Blue and DiI from the contralateral visual cortex. After the Fast Blue component of the tracer had been visualized in the LY-filter combination. the soma was impaled under visual control and LY was iontophoretically injected until all dendrites appeared brightly fluorescent. (A) When flling was completed the LY pipette was left in place. (B) Since the Fast Blue fluorescence had disappeared filters were switched to reveal the retrograde double-labelling with Dil. Thus erroneous impalement of neighbouring neurons was avoided. (C) After glycerol embedding the pyramidal cell was graphically reconstructed from serial negatives. Dendritic filling is extensive whereas axonal staining is poor. (D) At the same magnification the morphology of the Dil labelled soma exactly correlates with the graphical display. The origin of the four primary dendrites is indicated with arrows. Scaje in (A) and (B): $100 \mu \mathrm{m}$; in (C) and (D): $50 \mu \mathrm{m}$. 


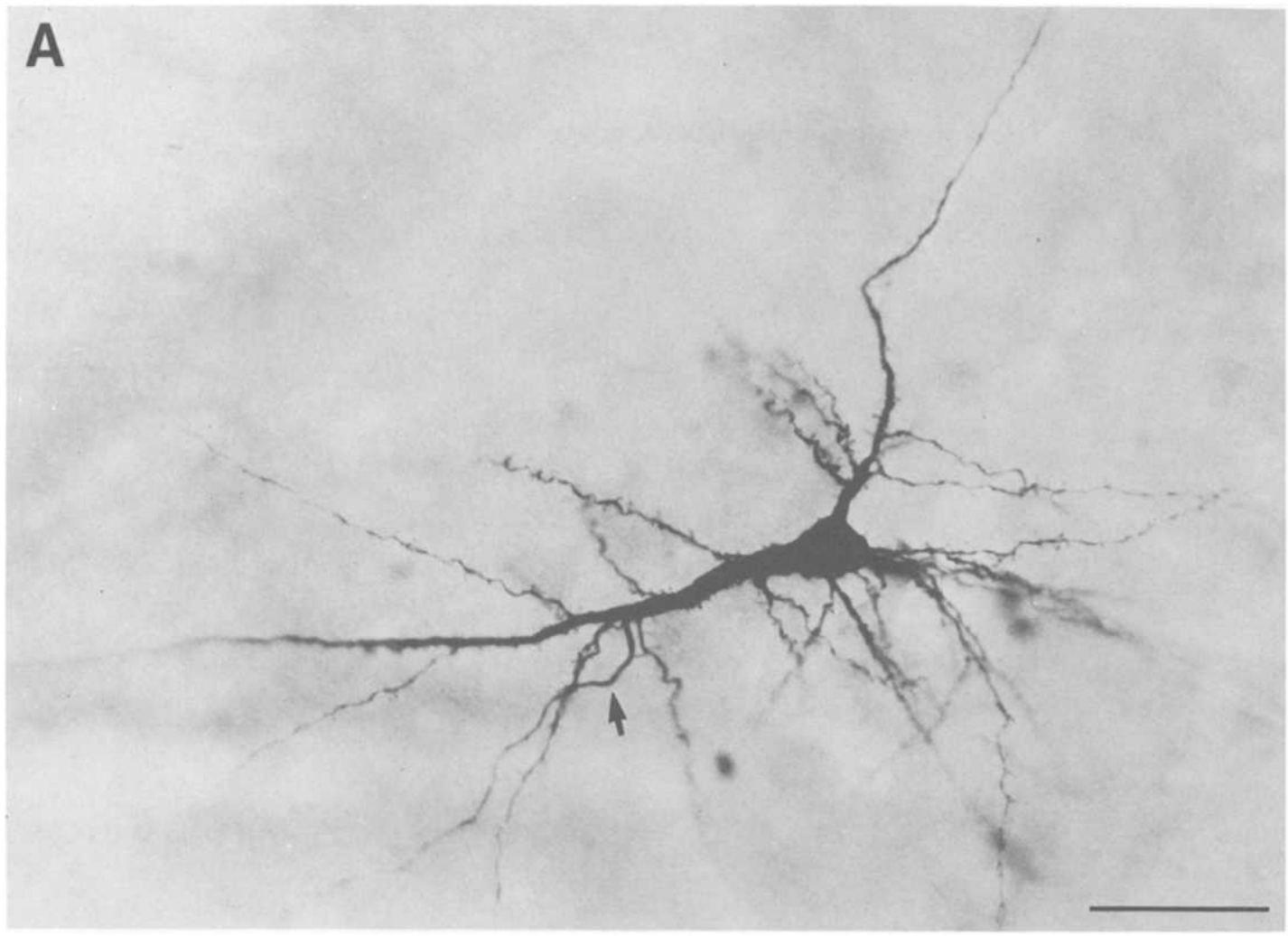

B

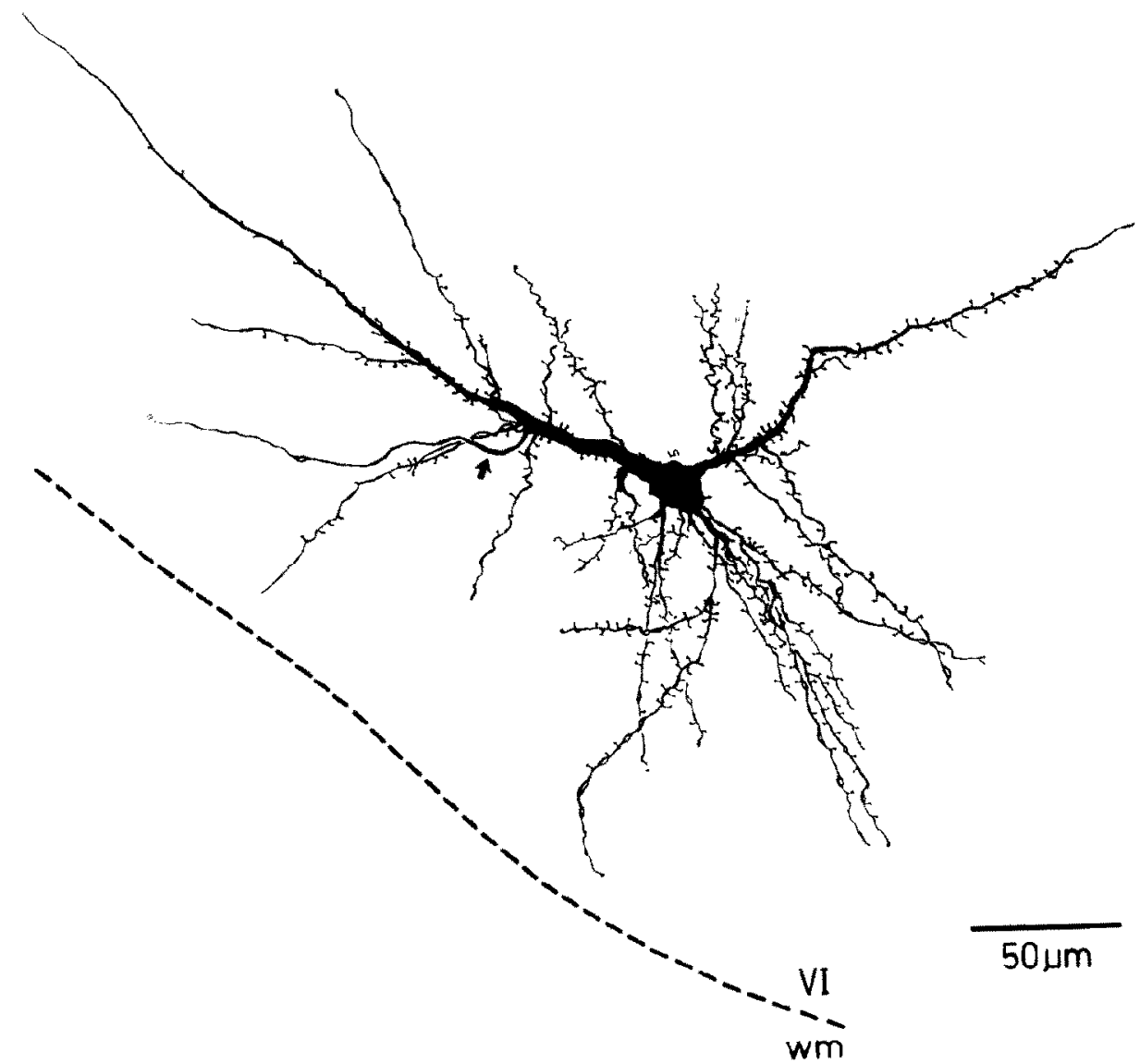

Fig. 4. (A) Micrograph of intracellular stained and photo-oxidized layer VIb pyramidal cell in area 17 of cat visual cortex (PN 19). The complete dendritic tree is homogeneously filled with the dark LY-DAB reaction product. Thus the appearance of photo-converted cells is similar to Golgi-impregnated neurons. A characteristic feature of many layer VI pyramidal cells is their horizontal orientation with respect to the pial surface. The axon (arrow) leaves the apical dendrite and descends towards the white matter. (B) Camera lucida reconstruction of the same neuron. A substantial number of ring-like varicosities in the basal dendrites indicates ongoing dendritic growth. The stippled line demarcates the border between layer VI and the white matter (wm). Scale in (A) and (B): $50 \mu \mathrm{m}$ 

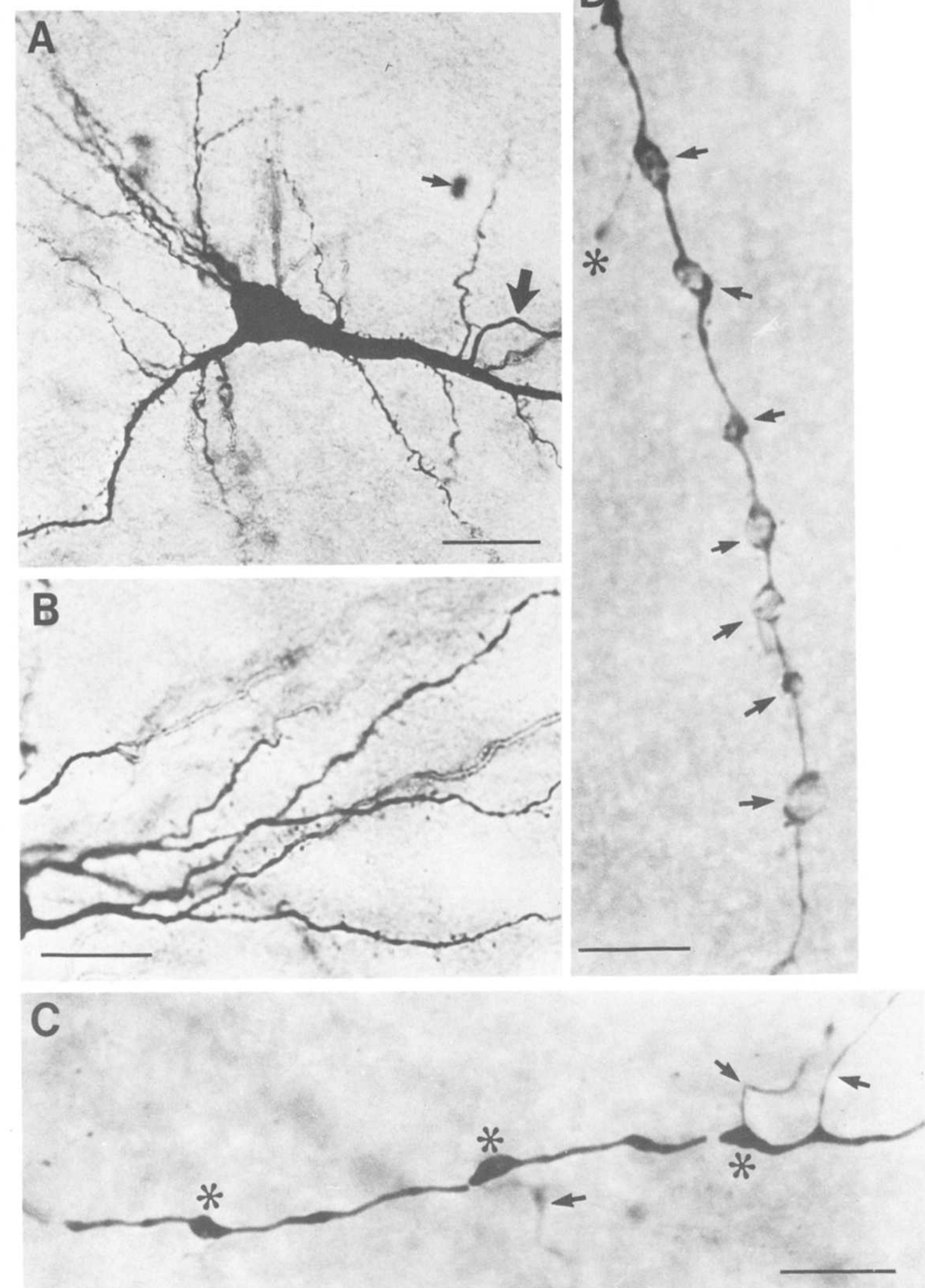

Fig. 5. Photographic details of LY-stained layer VI pyramidal cells in cat primary visual cortex after photo-oxidation. (A) Same cell as in Fig. $4 A$ at higher magnification which clearly reveals the spine morphology and distribution along the dendrites. The axon (large arrow) can be readily identified by its course and the absence of spines. Due to its endogenous peroxidase activity a slightly defocussed erythrocyte can be recognized (small arrow). The granular background staining results from the brief osmication procedure. (B) Detail of basal dendrites which are densely covered with spines. (C) Axon with three varicosities (asterisks) and three collaterals (arrows). (D) Basal dendrite of a layer VIa pyrimidal neuron (PN 5) with numerous large varicosities (arrows). A short process is emerging from the dendrite and ends in a growth cone-like structure (asterisk). Scale in (A): $25 \mu \mathrm{m}$ : in (B) (D): $10 \mu \mathrm{m}$. 


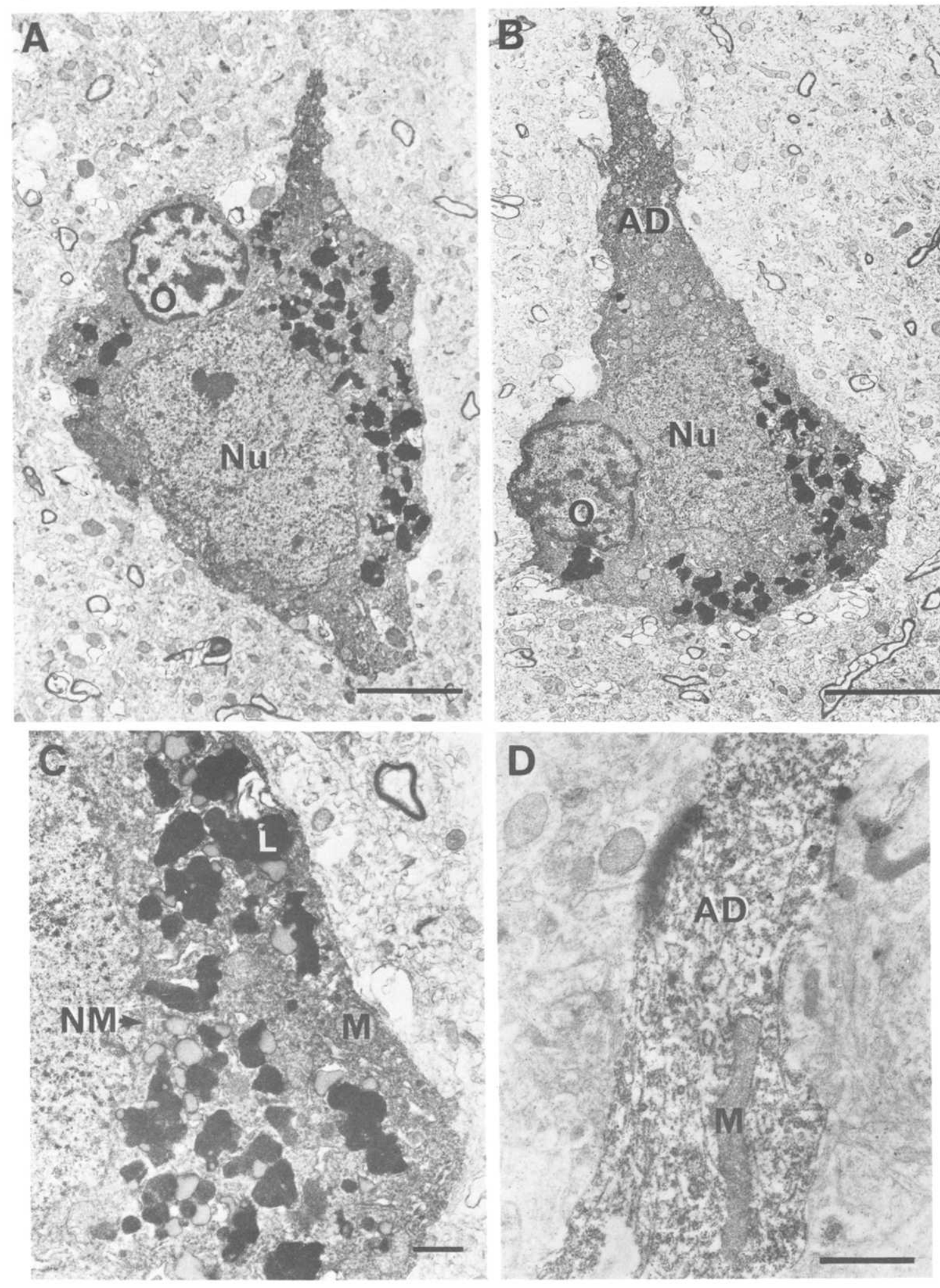

Fig. 6. Electron micrographs of LY-filled pyramidal cells after photo-oxidation and plastic embedding (A, B) Two layer $V$ pyramidal cells in human cortex can be readily discerned due to their opaque, fine granular reaction product, which is homogeneously distributed throughout the entire perikaryon. Both nuclei $(\mathrm{Nu})$ are surrounded by a rim of strongly contrasted lipofuscin granules $(\mathrm{L})$. Due to the strong autofiuorescence of the pigment part the age pigment had become photo-converted. Two satellite oligodendrocytes $(\mathrm{O})$ are attached to the pyramidal cell bodies. (C) Higher magnification of (A). Cytological details like mitochondria (M), the nuclear membrane (NM), lipid droplets and pigment granules $(\mathrm{L})$ become apparent. Despite poor tissue preservation there is no obvious leakage of reaction product into the extracellular space. (D) Apical dendrite of layer VI pyramidal cell in cat primary visual cortex. Opaque reaction product has spread to the distal portion of the dendrite. Despite decreasing contrast towards the dendritic tips, stained profles stand out with sufficient contrast from the surrounding neuropil. Scales in (A) and (B): $5 \mu \mathrm{m}$; in (C) and (D): $1 \mu \mathrm{m}$. 
immediate vicinity of the neuron graphical reconstruction was greatly simplified.

As a consequence of the non-randomness of the method it was possible to selectively stain neurons at any desired location or cortical layer (Fig. 2A-D). Using the latter advantage even in small pieces of human biopsy tissue large numbers of well filled cells were obtained. In addition, storage of the excess material in the refrigerator for periods longer than a week increased the sample considerably. Altogether staining worked very reliably and reproducibly, even when the tissue was immersion fixed after considerable delay (Fig. 2). Furthermore, the method is applicable in a variety of tissues and species (such as cat, rat, guinea-pig and human material) and has been successfully employed in specimens ranging from birth to old age.

\section{Combination with retrograde tracing}

Slices of cat visual cortex contralateral to the injection of the tracer mixture (Fast Blue and DiI) contained retrogradely labelled neurons located at the area $17 / 18$ border. Only layer 5 was devoid of labelled profiles. Inspection of the slices in both filter combinations revealed that a large number of cortical cells contained Fast Blue and DiI in co-localization. As described in Experimental Procedures, the tracer Fast Blue revealed neurons for subsequent targeting at the excitation wavelengths for LY. After filling was completed (Fig. 3A) and the Fast Blue fluorescence had become undetectable switching the filter combinations revealed the retrograde granular red label of DiI (Fig. 3B,D). Graphical reconstruction of the same neuron revealed its pyramidal morphology (Fig. 3C). Due to the cell's position in upper layer II/III the apical dendrite is rather short and forms a small apical tuft within layer I. Three basal dendrites arise from the soma (Fig. 3C,D) and branch into secondary and tertiary dendrites that remain within layers II/III. All higher order dendrites were covered with spines. Only one ascending axon collateral emerged from the main axon (arrow) which coursed towards the white matter

\section{Usefulness for light microscopy}

Initial attempts to analyse intracellularly stained and photo-oxidized material without osmium intensification were unsatisfactory. Even cells that had been intensively stained prior to bleaching had become only moderately brown after the conversion. Fine details like varicosities or spines had poor contrast and were difficult to resolve. This phenomenon is probably related to the high fluorescence yield of LY that is achieved by relatively small quanta of the dye ${ }^{69}$ Brief osmication ( $0.5 \%$ for $\left.1-5 \mathrm{~min}\right)$, however, resulted in a maximal gain of contrast resulting in an intensely black labelling of the complete dendritic tree (Fig. 3A). Further osmication only led to enhanced background staining.
So far, more than 200 pyramidal cells in developing cat visual cortex were filled, photo-converted and after osmication drawn with the aid of a camera lucida (Fig. 4b). In contrast to Golgi-preparations the vicinity of neurons was devoid of other impregnated elements obscuring the optics (Fig. 3A). Even prolonged times of light exposure over many hours did not bleach the DAB reaction product. Higher magnification revealed intensive Golgi-like staining of features as delicate as spines and dendritic varicosities (Fig. 5A,B,D). The material provided a basis for quantitative measurements of ontogenetic changes in neuronal morphology. ${ }^{45}$

\section{Combination with electron microscopy}

In order to correlate the dendritic morphology of neurons with their ultrastructure, human layer $V$ and several cat layer VI pyramidal cells underwent photooxidation after intracellular staining and subsequent graphical reconstruction. After plastic embedding, particularly after flat-embedding, ${ }^{28}$ the neurons and their position in the block were still discernible with the light microscope. Cut profiles of these pyramidal cells could be identified in $1 \mu \mathrm{m}$ semithin sections due to their dark reaction product. When cut profiles of these pyramids were identified, adjacent ultrathin sections were taken. Since the block contained only one individual neuron it was unnecessary to trace, in consecutive sections, the continuity of profiles containing reaction product. The latter was homogeneously distributed throughout the cell's cytoplasm and nucleus as a fine granular electron opaque label which extended into dendrites and the axon (Fig. $6 \mathrm{~A}-\mathrm{D})$. Due to the nature of the reaction product cytological details within the cellular boundaries were not obscured (Fig. 6C,D). Even in suboptimally fixed human material, the tissue preservation was sufficient to discern ultrastructural features, such as cytoplasmic organelles and membranes (Fig. 6A-C).

An interesting observation was made in respect to the age pigment lipofuscin which is abundant even in juvenile human cortex. The strongly autofluorescent pigment part of lipofuscin was photo-oxidized in the whole irradiated area in a similar fashion to LY. Therefore, the perinuclear rim of the age pigment granules appears electron opaque and strikingly dark in the electron microscopy micrographs (Fig. 6A-C).

\section{DISCUSSION}

The present paper details a variety of methodological aspects of intracellular LY injections in fixed brain slices. This technique is applicable in both perfusion- and immersion-fixed material ${ }^{8.57 .61}$ Thus, this staining approach may even be used for material, such as brain biopsies, where perfusion is impossible. ${ }^{*}$ In addition, the successful combination with light and electron microscopy and retrograde tracing has been demonstrated for three different experimental paradigms. Below, the properties, advantages and disad- 
vantages of LY-filling in fixed slices shall be discussed in relation to similar neuroanatomical approaches.

\section{Quality of staining}

The extent of dendritic staining which can be obtained by LY-filling in fixed slices appears to be virtually complete. Dye was observed in fine terminal dendrites, spines and dendritic varicosities. Comparison with published Golgi-data on mammalian visual cortex confirms the impression raised above. ${ }^{4,32,45,48,76}$ In the early postnatal period (PN 1-PN 11) dendritic growth cones of neurons in cat visual cortex were observed. As in most Golgi preparations a certain amount of the dendritic arbor was not contained within the tissue slice. However, since the majority of profiles leaving the plane of section was running in or close to the orthogonal plane the option of thicker slices was not considered as crucial for two-dimensional displays.

In contrast to extensive dendritic staining the spread of LY into axonal collaterals was rather poor. Occasionally local axon branches could be traced for distances of up to several hundred microns but termination sites were rarely observed. However, the latter disadvantage is also known for various Golgi stains which fail to impregnate the myelinated parts of the axonal arborization. ${ }^{47}$ Additionally, due to their capricious and random nature, Golgi impregnations may not reveal the morphology of rarely encountered neuronal types. In Golgi preparations other stained elements such as blood vessels, glia, fibres and surrounding nerve cells frequently obscure the optics whereas LY-filling mostly results in individually stained neurons.

Probably intracellular iontophoresis of HRP in vivo provides the most complete axonal filling and is superior to results obtained in fixed tissue. . $5,25,31,50,63$ However, the sample of neurons recovered from one animal is very low and intracellular impalement is biased towards frequently occurring cell types with large soma sizes. ${ }^{23}$ In addition, the range of possible combinations to increase the selectivity of sampling, for example antidromic activation from projection areas, ${ }^{59}$ is limited.

\section{Comparison with in vitro slice preparations}

Living slice preparations are mainly used for electrophysiological and pharmacological studies which require comparatively elaborate and sophisticated technical facilities ${ }^{13,14}$ The yield of stained neurons is rather low, particularly since living slices can be maintained only for several hours under in vitro conditions. Therefore, if the investigators interest is focussed mainly on anatomical questions, fixed slice preparations are a promising alternative. Firstly, recording equipment is not required and the material can be used for a considerable time, thus providing a basis for quantitative studies with samples exceeding 500 stained neurons. ${ }^{17}$ Secondly, the optical control under epifuorescent illumination permits the combination with other neuroanatomical tracing methods. ${ }^{60}$

Due to the apparently different properties of both methods the questions that might be answered with any of these technical approaches have rather little overlap. Therefore intracellular staining in fixed tissue is by no means considered as an alternative to in vitro preparations but rather as a new, powerful neuroanatomical staining method.

\section{Selectivity of staining}

Retrograde tracing with HRP or fuorescent dyes only rarely reveals the complete dendritic morphology of projection neurons. Therefore tracttracing with $\mathrm{HRP}^{22,65,67}$ and fluorescent retrograde labelling ${ }^{9}$ have been previously combined with Golgi impregnation. Due to the optical density of the Golgi precipitate the retrograde tracer only becomes visible after gold-toning, unless the neuron is partially impregnated. ${ }^{9,22,655,67}$ Both methods critically depend on the unpredictability of the Golgi method, in particular on the impregnation quality and the sampling bias of some neuronal types. ${ }^{22}$ Thus, there is always some uncertainty as to whether this combination reveals a representative sample of projection neurons.

In contrast, filling with LY in both fixed and living tissue $^{34}$ under visual control provides a high degree of selectivity. The technique may be used in combination with in vivo uptake of fluorescent transmitter analogues, ${ }^{58.72 .77}$ retrograde tracing, ${ }^{6.7,16,33,34,60}$ naturally occurring fluorescent markers such as the autofluorescent age pigment lipofuscin, ${ }^{8}$ or simply by employing fluorescent counterstains like acridine orange or DAPI. Since after filling retrogradely labelled cells the bright fluorescence of LY usually obscures the presence of other dyes with similar excitation wavelengths, some uncertainty may remain with respect to the neuron's double labelled nature. Therefore, the above approach of retrograde tracing with a mixture of two dyes fluorescing in different filter combinations allows the unequivocal identification of one of the transported dyes after LY-filling.

\section{Electron microscopy}

Due to the absence of a heavy metal atom, LY itself possesses no electron density. Therefore, it had previously been necessary to correlate adjacent thin sections prior to the analysis of ultrathin sections in the electron microscope ${ }^{70}$ Since even brief exposure to osmium tetroxide quenches the Lucifer fluorescence the material had to be stained in hafnium tetrachloride. ${ }^{70}$

Recently a much simpler and direct procedure to reveal the ultrastructure of $L Y$-stained neurons was developed. ${ }^{49}$ To increase the amount of reaction product photo-oxidation was performed in vivo although previous data indicated ultrastructural damages in LY-filled living tissue after ultraviolet illumination. ${ }^{51}$ Nevertheless, the fine granular nature of the reaction product revealed a wealth of cytological 
details within stained neurons. ${ }^{49,79}$ Despite marginally worse tissue quality, due to the absence of glutaraldehyde in the fixative, the results obtained in fixed slice preparations were of similar quality.

In comparison, material stained intracellularly with HRP and processed for electron microscopy reveals similar characteristics of the reaction product which is also homogeneously distributed in the entire cell without obscuring the fine anatomy of the neuron..$^{31,36.38 .66}$ However, the diffusion of the reaction product into the synaptic cleft ${ }^{37}$ or poor ultrastructural preservation of postsynaptic elements ${ }^{24}$ may occasionally lead to difficulties in the interpretation of the results.

The ultrastructural characteristics of the LY-DAB reaction product have considerable advantages over conventional Golgi-electron microscopic procedures, where the electron opacity of the Golgi deposit obscures the organelles of impregnated neurons. ${ }^{11,30,41,42,61,64,68,78}$ In addition, the silver chromate deposit frequently causes tears during ultrathin sectioning. ${ }^{20}$ However, Golgi-electron microscopic procedures where the silver precipitate is substituted by colloidal gold provide excellent ultrastructure of both deimpregnated neuron and surrounding tissue..$^{19.21 .52,53}$ Nevertheless, the latter procedure is still based on random impregnation and requires considerably more technical steps than the LY-DAB protocol.

\section{Future prospects of the method}

The present paper has emphasized three different possible applications of the LY technique in fixed slice preparations. One of the possible future implications is the combination with a variety of fluorescent tracers which can be used in both the retrograde and anterograde directions. Since it is possible to reliably discriminate three different tracer substances within one labelled cell body (e.g. Fast Blue, DiI, Diamidino Yellow), the morphology of neurons with three identified projection areas may be revealed by LY-injection.

Recently the combination of LY injections with immunocytochemistry has been reported. ${ }^{35.56 .62}$ Therefore, it should be possible to analyse the pharmacological input, particularly on the distal dendrites of identified projection neurons. After in vivo or in vitro high affinity uptake of fluorescent markers, that specifically label immunohistochemically defined subsets of neurons, ${ }^{58,72,77} \mathrm{LY}$ injection provides the basis for morphological identification and subsequent study of transmitter-specific synaptic connections.

At the ultrastructural level anterograde degeneration is commonly used in combination with retrograde or intracellular tracing. ${ }^{65}$ Anterograde degeneration in conjuction with retrograde tracing and LY injection may lead to experimental paradigms to unravel three neuronal chains or disynaptic pathways, respectively.

Acknowledgements - We are grateful to Profs. O. D. Creutzfeld, W. Singer and $\mathbf{H}$. Wässle for providing the technical facilities. The contribution of Prof. W. Schlote to the electron microscopy of the human material is gratefully acknowledged. Sincere thanks to Drs K. Albus and J. F. Dann for helpful discussions and critically reading the manuscript. This study was supported by grants of the Deutsche Forschungsgemeinschaft.

\section{REFERENCES}

1. Andrew R. D., MacVicar B. A., Dudek F. E. and Hatton G. I. (1981) Dye transfer through gap junctions between neuroendocrine cells of rat hypothalamus. Science 211, 1187-1189.

2. Antal M., Kraftsik R., Székely G. and Van der Loos H. (1986) Distal dendrites of frog motor neurons: a computer-aided electron microscopic study of cobalt-filled cells. J. Neurocytol. 15, 303-310.

3. Bentivoglio M., Kuypers H. G. J. M., Catsman-Berrevoets C. E., Loewe H. and Dann O. (1980) Two new fluorescent neuronal tracers which are transported over long distances. Neurosci. Lett. 18, 25-30.

4. Braak E. (1982) On The Structure of the Human Striate Area. Springer, Berlin.

5. Brown K. T. and Flaming D. G. (1986) Advanced Micropipette Techniques for Cell Physiology. John Wiley \& Sons, Chichester.

6. Buhl E. H. and Dann J. F. (1988) Morphological diversity of displaced retinal ganglion cells: a Lucifer Yellow study. J. comp. Neurol. 269, 210-218.

7. Buhl E. H. and Peichl L. (1986) Morphology of rabbit retinal ganglion cells projecting to the medial terminal nucleus of the accessory optic system. I. comp. Neurol. 253, 163-174.

8. Buhl E. H. and Schlote W. (1987) Intracellular Lucifer Yellow staining and electron microscopy of neurones in slices of fixed epitumourous human cortical tissue. Acta neuropath. 75, 140-146.

9. Catsicas S., Berbel P. J. and Innocenti G. M. (1986) A combination of Golgi impregnation and fluorescent retrograde labeling. J. Neurosci. Meth. 18, 325-332.

10. Catsicas S., Catsicas M. and Clarke P. G. H. (1987) Long-distance intraretinal connections in birds. Nature 326, 186-187.

11. Chan-Palay V. and Palay S. L. (1972) High voltage electron microscopy of rapid Golgi preparations. Neurons and their processes in the cerebellar cortex of monkey and rat. Z. Anat. EntwGesch. 139, 115-117.

12. Cobbett P. and Hatton G. I. (1984) Dye coupling in hypothalamic slices: dependence on in vivo hydration state and osmolarity of incubation medium. $I$. Neurosci. 4, 3034-3038.

13. Crunelli V., Leresche N., Hynd J. W., Patel N. M. and Parnavelas J. G. (1987) An in vitro slice preparation of the cat lateral geniculate nucleus. J. Neurosci. Meth. 20, 211-219.

14. Crunelli V., Leresche N. and Parnavelas J. G. (1987) Membrane properties of morphologically identified X and Y cells in the lateral geniculate nucleus of the cat in vitro. J. Physiol. 390, 243-256.

15. Cullheim S. and Kellerth J.-O. (1976) Combined light and electron microscopic tracing of neurons, including axons and synaptic terminals, after intracellular injection of horseradish peroxidase. Neurosci. Lett. 2, 207-213. 
16. Dann J. F. and Buhl E. H. (1987) Rat retinal ganglion cells projecting to the accessory optic system. J. comp. Neurol. 262, 141-158.

17. Dann J. F., Buhl E. H. and Peichl L. (1987) Dendritic maturation in cat retinal ganglion cells: a Lucifer Yellow study. Neurosci. Lett. 80, 21-26.

18. Davis N. T. (1982) Improved methods for cobalt filling and silver intensification of insect motor neurons. Stain Technol. 57, 239-244.

19. DeFelipe J. and Fairén A. (1982) A type of basket cell in superficial layers of the cat visual cortex. A Golgi-electron microscope study. Brain Res. 244, 9-16.

20. Fairén A., Peters A. and Saldanha J. (1977) A new procedure for examining Golgi impregnated neurons by light and electron microscopy. J. Neurocytol. 6, 311-337.

21. Fairén A. and Valverde F. (1980) A specialized type of neuron in the visual cortex of cat. A Golgi and electron microscopic study of chandelier cells. J. comp. Neurol. 194, 761-779.

22. Freund T. F. and Somogyi P. (1983) The section-Golgi impregnation procedure. I. Description of the method and its combination with histochemistry after intracellular iontophoresis or retrograde transport of horseradish peroxidase. Neuroscience 9, 463-474.

23. Friedlander M. J., Lin C.-S., Stanford L. R. and Sherman M. S. (1981) Morphology of functionally identified neurons in lateral geniculate nucleus of the cat. J. Neurophysiol. 46, 80-129.

24. Gabbott P. L. A., Martin K. A. C. and Whitteridge D. (1987) Connections between pyramidal neurons in layer 5 of cat visual cortex (area 17). J. comp. Neurol. 259, 364-381.

25. Gilbert C. D. and Wiesel T. N. (1979) Morphology and intracortical projections of functionally characterised neurones in the cat visual cortex. Nature 280, 120-125.

26. Gilbert C. D. and Wiesel T. N. (1983) Clustered intrinsic connections in cat visual cortex. J. Neurosci. 3, 11161133.

27. Gutnick M. J. and Prince D. A. (1981) Dye coupling and possible electrotonic coupling in the guinea pig neocortical slice. Science 211, 67-70.

28. Holländer H. (1970) The section embedding (SE) technique: a new method for the combined light microscopic and electron microscopic examination of central nervous tissue. Brain Res. 20, 39-47.

29. Honig M. G. and Hume R. I. (1986) Fluorescent carbocyanine dyes allow living neurons of identified origin to be studied in long-term cultures. J. Cell Biol. 103, 171-187.

30. Ito H. and Atencio F. (1976) Staining methods for an electron microscopic analysis of Golgi impregnated nervous tissue and a demonstration of the synaptic distribution upon pulvinar neurons. J. Neurocytol. 5, 297-317.

31. Jankowska E., Rastad J. and Westman J. (1976) Intracellular application of horseradish peroxidase and its light and electron microscopic appearance in spinocervical tract cells. Brain Res. 105, 557-562.

32. Jones E. G. (1975) Varictics and distribution of non-pyramidal cells in the somatic sensory cortex of the squirrel monkey. J. comp. Neurol. 160, 205-268.

33. Katz L. C. (1987) Local circuitry of identified projection neurons in cat visual cortex brain slices. J. Neurosci. 7, $1223-1249$.

34. Katz L. C., Burkhalter A. and Dreyer W. J. (1984) Fluorescent latex microspheres as a retrograde neuronal marker for in vivo and in vitro studies of visual cortex. Nature 310, 498-500.

35. Kayser B. E. J., Muhlethaler M. and Dreifuss J. J. (1982) Paraventricular neurons in the rat hypothalamic slice: Lucifer yellow injections and immunocytochemical identification. Experientia 38, 391-393.

36. Kisvarday Z. F., Martin K. A. C., Freund T. F., Magloczky Zs., Whitteridge D. and Somogyi P. (1986) Synaptic targets of HRP-filled layer III pyramidal cells in the cat striate cortex. Expl Brain Res. 64, 541-552.

37. Kisvarday Z. F., Martin K. A. C., Friedlander M. J. and Somogyi P. (1987) Evidence for interlaminar inhibitory circuits in the striate cortex of the cat. J. comp. Neurol. 260, 1-19.

38. Kisvarday Z. F., Martin K. A. C., Whitteridge D. and Somogyi P. (1985) Synaptic connections of intracellularly filled clutch cells: a type of small basket cell in the visual cortex of the cat. J. comp. Neurol. 241, 111-137.

39. Kitai S. T., Kocsis J. D., Preston R. J. and Sugimori M. (1976) Monosynaptic inputs to caudate neurons identified by intracellular injection of horseradish peroxidase. Brain Res. 109, 601-606.

40. Knowles W. D., Funch P. G. and Schwartzkroin P. A. (1982) Electrotonic and dye coupling in hippocampal CA1 pyramidal cells in vitro. Neuroscience 7, 1713-1722.

41. Kolb H. (1974) The connection between horizontal cells and photoreceptors in the retina of the cat: electron microscopy of Golgi preparations. J. comp. Neurol. 155, 1-14.

42. LeVay S. (1973) Synaptic patterns in the visual cortex of the cat and monkey. Electron microscopy of Golgi preparations. J. comp. Neurol. 150, 53-86.

43. Light A. R. and Durkovic R. C. (1976) Horseradish peroxidase: an improvement in intracellular staining of single, electrophysiologically characterized neurons. Expl Neurol. 53, 847-853.

44. Lin C. S., Friedlander M. J. and Sherman S. M. (1979) Morphology of physiologically identified neurons in the visual cortex of the cat. Brain Res. 172, 344-349.

45. Lübke J. (1987) Postnatale Entwicklung von Schicht VI-Pyramidenneuronen im visuellen Cortex der Katze (Felis domestica). Untersuchungen am fixierten Slice. Diplom-thesis in Zoology, University of Göttingen.

46. Lund J. S. (1973) Organization of neurons in the visual cortex, area 17, of the monkey (Macaca mulatta). J. comp. Neurol. 147, 455-496.

47. Lund J. S. (1987) Local circuit neurons of macaque monkey striate cortex: I. Neurons of laminae $4 \mathrm{C}$ and $5 \mathrm{~A}$. J. comp. Neurol. 257, 60-92.

48. Lund J. S. and Boothe R. (1975) Interlaminar connections and pyramidal neuron organization in the visual cortex, area 17 , of the macaque monkey. J. comp. Neurol. 159, 305-334.

49. Maranto A. R. (1982) Neuronal mapping: a photooxidation reaction makes Lucifer Yellow useful for electron microscopy. Science 217, 953-955.

50. Martin K. A. C., Somogyi P. and Whitteridge D. (1983) Physiological and morphological properties of identified basket cells in the cat's visual cortex. Expl Brain Res. 50, 193-200.

51. Miller J. P. and Selverston A. I. (1979) Rapid killing of single neurons by irradiation of intracellularly injected dye. Science 206, 702-704. 
52. Peters A. and Fairen A. (1978) Smooth and sparsely-spined stellate cells in the visual cortex of the rat: a study using a combined Golgi-electron microscope technique. J. comp. Neurol. 181, 129-172.

53. Peters A. and Proskauer C. C. (1980) Synaptic relationships between a multipolar stellate cell and a pyramidal neuron in the rat visual cortex. A combined Golgi-electron microscopic study. J. Neurocytol. 9, 163-183.

54. Pitman R. M., Tweedle C. D. and Cohen M. J. (1972) Branching of central neurons: intracellular cobalt injection for light and electron microscopy. Science 176, 412-414.

55. Rao G., Barnes C. A. and McNaughton B. L. (1986) Intracellular fluorescent staining with carboxyfluorescein: a rapid and rcliable method for quantifying dyc-coupling in mammalian central nervous system. J. Neurosci. Meth. 16, 251263.

56. Reaves T. A., Hou-Yu, Zimmerman E. A. and Hayward J. N. (1983) Supraoptic neurons in the rat hypothalamoneurohypophysial explant: double-labelling with Lucifer yellow injection and immunocytochemical identification of vasopressin- and neurophysin-containing neuroendocrine cells. Neurosci. Lett. 37, 137-142.

57. Rho J.-H. and Sidman R. L. (1986) Intracellular injection of Lucifer Yellow into lightly fixed cerebellar neurons. Neurosci. Lett. 72, 21-24.

58. Sandell J. H. and Masland R. H. (1986) A system of indoleamine-accumulating neurons in the rabbit retina. J. Neurosci. 6, 3331-3347.

59. Schofield B. R., Hallman L. E. and Lin C.-S. (1987) Morphology of corticotectal cells in the primary visual cortex of hooded rats. J. comp. Neurol. 261, 85-97.

60. Schwerdtfeger W. K. and Buhl E. (1986) Various types of non-pyramidal hippocampal neurons project to the septum and contralateral hippocampus. Brain Res. 386, 146-154.

61. Scott G. L. and Guillery R. W. (1974) Studies with the high voltage electron microscope of normal, degenerating, and Golgi impregnated neuronal processes. J. Neurocytol. 3, 567--590.

62. Smithson K. G., Cobbett P., MacVicar B. A. and Hatton G. I. (1984) A reliable method for immunocytochemical identification of Lucifer Yellow injected, peptide containing mammalian central neurons. J. Neurosci. Meth. 10, 59-69.

63. Snow P. J., Rose P. K. and Brown A. G. (1976) Tracing axons and axon collaterals of spinal neurons using intracellular injection of horseradish peroxidase. Science 191, 312-313.

64. Somogyi P. and Cowey A. (1984) Double bouquet cells. In Cerebral Cortex (eds Peters A. and Jones E. G.), Vol. 1, pp. 337 360. Plenum Press, New York.

65. Somogyi P., Hodgson A. J. and Smith A. D. (1979) An approach to tracing neuron networks in the cerebral cortex and basal ganglia. Combination of Golgi staining, retrograde transport of horseradish peroxidase and anterograde degeneration of synaptic boutons in the same material. Neuroscience 4, 1805-1852.

66. Somogyi P., Kisvárday Z. F., Martin K. A. C. and Whitteridge D. (1983) Synaptic connections of morphologically identified and physiologically characterised large basket cells in the striate cortex of cat. Neuroscience 10, 261-294.

67. Somogyi P. and Smith A. D. (1979) Projection of neostriatal spiny neurons to the substantia nigra. Application of a combined Golgi-staining and horseradish peroxidase transport procedure at hoth light-and electron-micrnscopic levels. Brain Res. 178, 315.

68. Stell W. K. (1965) Correlation of retinal cytoarchitecture and ultrastructure in Golgi preparations. Anat. Rec. 153, 389-397.

69. Stewart W. W. (1978) Functional connections between cells as revealed by dye-coupling with a highly fluorescent naphthalimide tracer. Cell 14, 741-759.

70. Stewart W. W. (1981) Lucifer dyes-highly fluorescent dyes for biological tracing. Nature 292, 17-21.

71. Stretton A. O. and Kravitz E. A. (1968) Neuronal geometry: determination with a technique of intracellular dye injection. Science 162, 132-134.

72. Tauchi M. and Masland R. H. (1984) The shape and arrangement of the cholinergic neurons in the rabbit retina. Proc. R. Soc. Lond. (Biol.) 223, 101-119.

73. Tauchi M. and Masiand R. H. (1985) Local order among the dendrites of an amacrine cell population. J. Neurosci. 5, 2494-2501.

74. Thanos S. and Bonhoeffer F. (1987) Axonal arborization in the developing chick retinotectal system. J. comp. Neurol. 261, $155-164$.

75. Tusa R. J., Palmer L. A. and Rosenquist A. C. (1978) The retinotopic organization of area 17 (striate cortex) in the cat. J. comp. Neurol. 177, 213-236.

76. Valverde F. (1978) The organization of area 18 in the monkey: a Golgi study. Anat. Embryol. 154, $305-334$.

77. Vaney D. I. (1986) Morphological identification of serotonin accumulating neurons in the living retina. Science 233, 444-446.

78. West R. W. (1976) Light and electron microscopy of the ground squirrel retina: functional considerations. $J$. comp. Neurol. 168, 355-378.

79. Zimmerman R. P. (1986) Specific neuronal staining by in vitro uptake of Lucifer Yellow. Brain Res. 383, $287-298$.

(Accepted 25 May 1988) 